QUARTERLY OF APPLIED MATHEMATICS

VOLUME LXVIII, NUMBER 3

SEPTEMBER 2010, PAGES 591-606

S $0033-569 X(2010) 01174-3$

Article electronically published on May 19, 2010

\title{
ON DIFFUSIVE SLOWDOWN IN THREE-LAYER HELE-SHAW FLOWS
}

\author{
$\mathrm{BY}$ \\ PRABIR DARIPA (Department of Mathematics, Texas A\&M University, College Station, Texas \\ $77843)$ \\ AND \\ GELU PASA (Institute of Mathematics "Simion Stoillow" of Romanian Academy, Bucharest, \\ Romania 70700)
}

Abstract. In a recently published article of Daripa and Pasa [Transp. Porous Media (2007) 70:11-23], the stabilizing effect of diffusion in three-layer Hele-Shaw flows was proved using an exact analysis of normal modes. In particular, this was established from an upper bound on the growth rate of instabilities which was derived from analyzing stability equations. However, the method used there is not constructive in the sense that the upper bound derived from actual numerical discretization of the problem could be significantly different from the exact one reported depending on the scheme used. In this paper, a numerical approach to solve the stability equations using a finite difference scheme is presented and analyzed. An upper bound on the growth rate is derived from numerical analysis of the discrete system which also shows the diffusive slowdown of instabilities. Upper bounds obtained by this numerical approach and by the analytical approach are compared. The present approach is constructive and directly leads to the implementation of the numerical approach to obtain approximate solutions in the presence of diffusion. The contributions of the paper are the novelty of the approach and a bound on the growth rates that does not depend on the solution itself.

1. Introduction. The subject of multi-layer (more than two-layer) multi-phase flows has received much less attention than similar two-layer flows, partly because of the level of difficulty associated with studies of such flows. Such flows are usually richer in complexity and involve many parameters and variables whose control and manipulations in innovative ways can prove to be useful technologically. To make theoretical and computational advances in this direction, the mathematical formulation of such problems is the first necessary step. One such problem that has received some attention ([1], 3]), partly motivated by industrial applications ([14, [17, [23], 25], 28]), involves three-layer

Received February 1, 2009.

2000 Mathematics Subject Classification. Primary 76E17, 76T30, 76R50, 65F99, 65Q05.

E-mail address: daripa@math.tamu.edu

E-mail address: Gelu.Pasa@imar.ro

(C)2010 Brown University 
immiscible flows in Hele-Shaw cells involving two sharp interfaces initially. The physical setup of the problem involves three layers of fluid in a Hele-Shaw cell with the least viscous fluid such as water displacing an intermediate layer of fluid having variable viscosity. This layer in turn displaces the most viscous fluid such as oil. The fluid in the intermediate layer is an aqueous phase consisting of a polymer in water. Pointwise viscosity of this aqueous phase (to be called 'poly-solution' henceforth) in this intermediate layer depends on the local concentration of the polymer. In our model, the polymer is passively advected by the fluid and therefore, the viscosity profile of the middle layer dynamically evolves with the velocity field in this region. The simplest nontrivial admissible solution of the underlying equations is the uniform motion of all three layers having two planar interfaces separating these three layers. The stability of this system has been addressed earlier (see [3], [5]) for arbitrary viscosity profiles in the intermediate layer.

In this paper, our model allows diffusion of the polymer in the above problem setup. In a recent paper, Daripa and Pasa ([6]) first mathematically formulated this problem incorporating diffusion of the polymer in the middle layer. In that paper, stability equations were derived in a form suitable for classical analysis using normal modes. The resulting eigenvalue problem was then analyzed using weak formulation in order to obtain quantitative results about the effect of diffusion on flow instability. Such results were obtained in the form of upper bounds on the growth rate. The present paper develops a constructive method to obtain similar bounds as in Daripa and Pasa [6] using numerical analysis. The method is constructive in the sense that the bounds are obtained by first developing a numerical scheme to solve the stability equations whose implementation and subsequent mining of numerical data to obtain approximate solutions will be taken up in the future. An interesting possibility is that there could be transient growth, because the original problem is not selfadjoint. This might change the conclusion that the polymeric diffusivity stabilizes the flow. A reference to transient growth and its applications is the book of Henningson and Schmidt.

In most fluid flows, molecular diffusion slows down the growth of instabilities except for some flows such as parallel shear flows. Such cannot be said, without analytical justification, of the role of polymer diffusion in this problem since its role is somewhat different from that of molecular diffusion in fluid flows. In the middle layer, instabilities create nonuniformities in polymer concentration, which in turn drives diffusion of the polymer, an effect neglected thus far in the literature. This diffusion of the polymer dynamically changes the local viscosity gradients in the middle layer and the magnitudes of jumps in viscosities across the interfaces which respectively drive the growth rates of instabilities in the middle layer and on two interfaces. The extent to which the growth of these instabilities is affected depends on the diffusion coefficient. Quantifying this effect in terms of the diffusion coefficient can be useful in providing an insight into the rough effect of diffusion on the flow. In this paper, we investigate these issues using numerical discretization of the underlying stability equations.

We obtain bounds on the growth rate using numerical analysis of the stability equations. The diffusion coefficient dependent bound obtained numerically clearly shows diffusive slowdown of instabilities which supports our results reported recently in Daripa and Pasa [6]. Since the method presented here relies on first developing a numerical 
scheme to solve the stability equations, the numerical scheme given here can be implemented, if so desired, to construct approximate solutions. This will allow one to explore numerically the effects of diffusion on the modal growth rates and eigenfunctions of the normal modes. We must stress that these numerical implementation issues are not the subject of this paper, though we intend to undertake this task in the future.

It is important to emphasize here that the purpose of the numerical analysis of the specific scheme carried out here is not so much as to just obtain a bound on a physical quantity (i.e., the maximal growth rate) but to ensure that the numerical scheme proposed for solving the stability equations has some desirable properties of the continuum equations that are useful to have before using the scheme to construct approximate solutions. Numerically estimating the bound and assuring that it compares reasonably well with the exact bound, which has been done in this paper, precisely serves that purpose. This scheme then should be useful in computing the early stages of evolution of initial disturbances. The evolution of interfacial disturbances based on this scheme during initial stages then can be used as a benchmark for the purpose of validating any numerical calculation for the full initial value problem from which linear stability equations are derived. This is another motivation for the work carried out in this paper.

Since three-layer Hele-Shaw flows with diffusion partly build upon two-layer HeleShaw flows, it is appropriate to add some relevant literature on various pertinent aspects of such two-layer flows, which we do next in this paragraph. There have been extensive experimental, theoretical and numerical works on two-layer immiscible as well as miscible Hele-Shaw flows. The literature is huge, and we can cite only a few here. The earliest theoretical works on viscosity-jump driven interfacial instability of such two-layer immiscible flows date back to the late fifties (see [21, [19] for Hele-Shaw flows and 2] for flows in permeable media). Subsequently, there have been many studies on such two-layer flows such as exact nontrivial solutions in the form of fingers with and without surface tension ([7, 27, [12, 29]), singularity formation (see [18]), numerical and perturbation studies (1], [16]), etc. For more exhaustive references on various numerical, experimental, and theoretical works, the review articles of Saffman (see [20]) and Kessler et al. (see [13]) are worth mentioning. Towards this end, works of Hickernell and Yortsos (see [8]), Yortsos and Zeybek (see [30]), Loggia et al. (see [15]), and Shariati and Yortsos (see 24]) on two-layer miscible flows should also be cited.

In closing this section, we comment on how this work on Hele-Shaw flows relates to porous media. In porous media, transport of reactive as well as nonreactive species occurs in a myriad of processes either naturally and/or artificially by design, e.g., transport of contaminants, transport of biomass and solutes, transport of various species during various oil recovery processes, just to mention a few. In modeling such porous media flow processes involving a variety of species, diffusion of species naturally plays a significant role. Therefore, assessing the effect of species diffusion on various flow processes including hydrodynamic stability is important. This is what we do in this paper for immiscible flows using the Hele-Shaw model as opposed to the more appropriate Buckley-Leverett model, but this compromise has an insignificant effect on the basic problem of diffusive slowdown we are addressing here. For example, the displacement of oil by water in porous media by the Buckley-Leverett model allows shock waves and rarefaction waves 
in saturation. A shock wave creates a jump in mobility across it, whereas the saturation waves behind the shock wave create a mobility gradient behind the shock. To a large extent, this is very well modeled using Hele-Shaw models instead with a sharp jump in viscosity (mobility) across the interface separating displaced and displacing fluids and a viscous profile behind the interface modeling the effect of rarefaction waves. The HeleShaw model that we consider below has all these properties.

\section{Preliminaries.}

2.1. Problem formulation. Figure 1 shows a three-layer immiscible flow in a Hele-Shaw cell with two planar interfaces moving uniformly at a constant velocity. Our goal in this paper is to provide a constructive approach to study the stability of this flow under a proper setting that takes into account the desired viscous profiles of these three layers and diffusion effects in the middle layer which are made precise below. As we will see, this study provides a precise estimate of the effect of diffusion on flow instabilities.

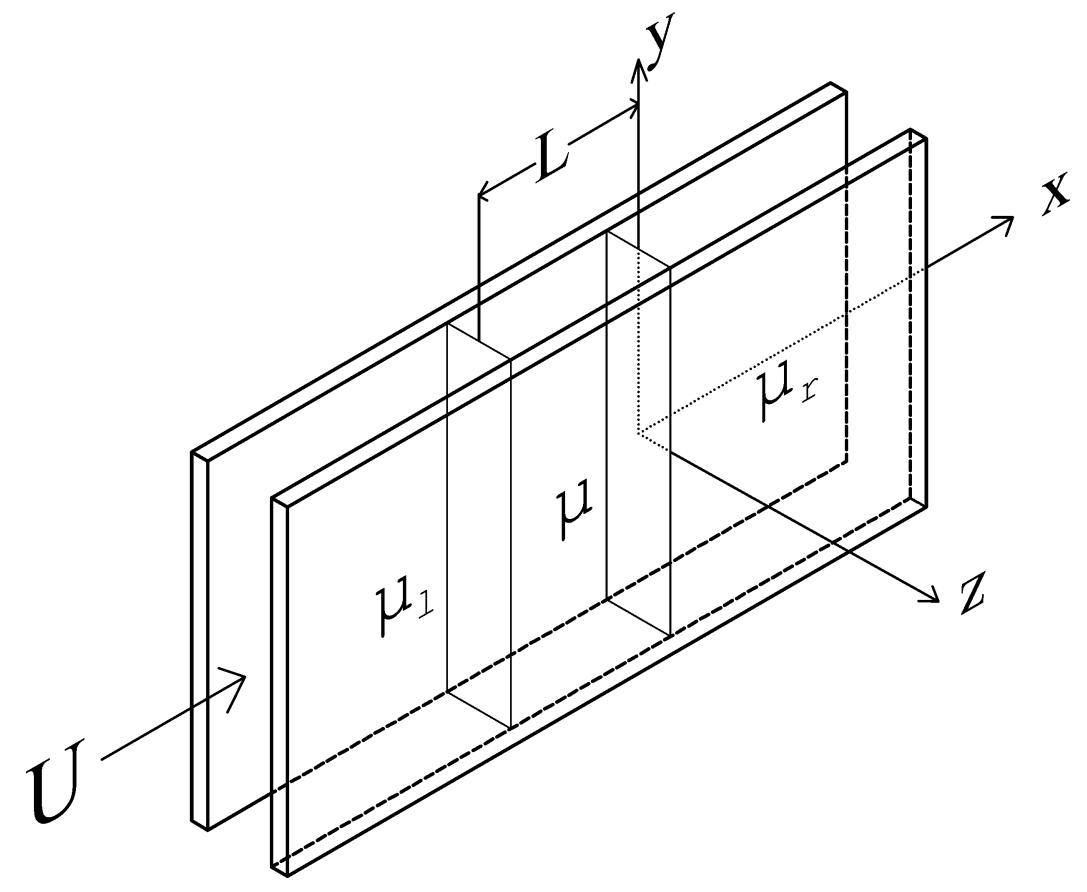

FIG. 1. Three-layer fluid flow in a Hele-Shaw cell

The flow in the Hele-Shaw cell is essentially two-dimensional: $\Omega:=(x, y)=\mathbb{R}^{2}$ with a periodic extension of the setup in the $y$-direction. The uniform velocity far upstream is $\mathbf{u}=(U, 0)$, and the fluid in between the middle layer of length $L$ has a smooth viscous profile $\mu(x)$ such that $\mu_{l}<\mu(x)<\mu_{r}$, where $\mu_{l}$ and $\mu_{r}$ are the viscosities of the fluids in the left and right extreme layers respectively (see Fig. 1). It is assumed that the middle layer has an aqueous phase which is polymer-thickened water (to be called 'polysolution' henceforth), and the viscosity $\mu$ of the poly-solution is an invertible function of 
the polymer concentration $c$. The polymer concentration profile $c(x)(-L<x<L)$ of the middle layer determines the viscous profile $\mu(x)$ of this layer.

The case of passive advection of the polymer by the fluid in the middle layer has been addressed earlier by several authors (see [11, 3]). Thus, in this model, the viscosity of every fluid element in the poly-solution is a constant of motion. In this paper, we break this invariance property by also allowing diffusion of the polymer in the middle layer, which is more realistic. Hitherto, this diffusion effect has not been addressed in this three-layer flow. The advection-diffusion equation of the polymer in the middle layer is governed by

$$
\frac{\partial c}{\partial t}+\mathbf{u} \cdot \nabla c=\eta \triangle c
$$

where $\eta$, the diffusion coefficient, is a constant. Since the viscosity $\mu$ is an invertible function of the concentration $c$, the same equation holds true in the middle layer for viscosity $\mu(x)$ in the middle layer. Therefore, the governing equations in each layer are

$$
\begin{aligned}
\boldsymbol{\nabla} \cdot \mathbf{u} & =0, \\
\boldsymbol{\nabla} p & =-\mu \mathbf{u}, \\
\frac{\partial \mu}{\partial t}+\mathbf{u} \cdot \nabla \mu & =\eta \triangle \mu,
\end{aligned}
$$

where $p$ is the pressure, $\nabla=\left(\frac{\partial}{\partial x}, \frac{\partial}{\partial y}\right)$, and $\triangle$ is the Laplacian in the plane. The first equation (2.1) is the continuity equation for incompressible flow, the second equation (2.2) is Darcy's law (Darcy (1856)), and the third equation (2.3) is the advection-diffusion equation for viscosity. Modeling such flows using Darcy's law (2.2) has been the practice in the literature for a long time, including the article of Taylor and Saffman (see [21]) which originally appeared in the journal: Proceedings of Royal Society. In using such a model, averaging is first done in the vertical direction in the original configuration shown in Fig. 1 and then the motion of the fluid is assumed to evolve as per the equation resulting from averaging, i.e. Darcy's law. Thus the parabolic profile in the $z$-direction separating two fluids does not arise, which is acceptable since the fluid on the walls is moving with the average velocity in the $z$-direction, which is not the physical velocity. This model has been accepted in thousands of articles starting with the experiments of Taylor. Taylor showed in physical experiments the development of fingers, which were also obtained theoretically using Darcy's law within a very good approximation by Saffman and Taylor [21]. There is a rich history to it in fluid mechanics. For example, see Saffman [20, Homsy [9], Tanveer [26] and Howison [10] just to mention a few. In short, this model does not include the structure of displacement in the gap between the plates.

The setup shown in Fig. 1 and discussed at the beginning of this section is a basic solution of the above system with the following qualifications: the extreme layer viscosities $\mu_{l}$ and $\mu_{r}$ are constants and the viscous profile of the middle layer is linear, with viscosity increasing in the direction of flow in this layer. The pressure corresponding to this basic solution can be obtained by integrating (2.2). It is convenient to work in a moving frame with velocity $(U, 0)$ so that the basic solution is stationary in this moving frame. Henceforth, our discussion including equations will be in the moving frame, unless 
otherwise mentioned. Here and below, with a slight abuse of notation, the same variable $x$ is used to refer to the $x$-coordinate in the moving reference frame.

2.2. Stability equations. In the moving frame, the basic state is $\left(u=0, v=0, p_{0}(x)\right.$, $\mu(x))$, where the basic viscous profile $\mu(x)$ of the middle layer $(-L<x<L)$ is linear, namely $\mu(x)=a x+b$, with viscosity increasing in the direction of flow from $\mu(-L)>\mu_{l}$ to $\mu(0)<\mu_{r}$ in this layer. If this basic state is perturbed by $(\epsilon \tilde{u}, \epsilon \tilde{v}, \epsilon \tilde{p}, \epsilon \tilde{\mu})$, where $\epsilon$ is a small parameter and the perturbed state $\left(\epsilon \tilde{u}, \epsilon \tilde{v}, p_{0}(x)+\epsilon \tilde{p}, \mu(x)+\epsilon \tilde{\mu}\right)$ is then substituted in the moving frame version of the equations (2.1) through (2.3), then we obtain the following linearized equations for $\tilde{\mathbf{u}}=(\tilde{u}, \tilde{v}), \tilde{p}$, and $\tilde{\mu}$ at the order $O(\epsilon)$ :

$$
\begin{array}{lr}
\nabla \cdot \tilde{\mathbf{u}}=0, & x, y \in \mathbb{R}, \\
\nabla \tilde{p}=-\mu \tilde{\mathbf{u}}-\tilde{\mu}(U, 0), & x, y \in \mathbb{R}, \\
\frac{\partial \tilde{\mu}}{\partial t}+\tilde{u} \frac{d \mu}{d x}=\eta \triangle \tilde{\mu}, & -L<x<0 .
\end{array}
$$

The above equations are studied by the method of normal modes in which a typical wave disturbance has the form

$$
(\tilde{u}, \tilde{v}, \tilde{p}, \tilde{\mu})=(f(x), \psi(x), \phi(x), h(x)) e^{(i k y+\sigma t)},
$$

where $k$ is a real axial wavenumber and $\sigma$ is the growth rate, which could be complex. Substitution of (2.7) in (2.4) through (2.6) results in equations involving $f(x), \psi(x), \phi(x)$, and $h(x)$ whose manipulation then leads to two coupled equations (see equations (2.8) and (2.8) 2 below) involving $f(x)$ and $h(x)$. These are subject to boundary conditions resulting from the linearization of the kinematic and the dynamic boundary conditions at two interfaces as derived in Daripa and Pasa [4] for the zero-diffusion case. These boundary conditions are independent of the diffusion process in the middle layer. Thus, in this model, where the viscosity is advected as well as diffused by the fluid in the middle layer, the evolution of linearized disturbances is governed by the following problem:

$$
\left.\begin{array}{l}
-\left(\mu f_{x}\right)_{x}+k^{2} \mu f=-k^{2} U h, \quad x \in(-L, 0), \\
\eta h_{x x}-\left(\sigma+\eta k^{2}\right) h=a f, \quad a>0, \quad x \in(-L, 0), \\
f_{x}(0)=(\lambda \mathcal{P}+q) f(0), \quad f_{x}(-L)=(\lambda r+s) f(-L), \\
h(0)=h(-L)=0
\end{array}\right\}
$$

where $\lambda=1 / \sigma, a=(\mu(0)-\mu(-L)) / L>0, \eta>0$, and $\mathcal{P}, q, r, s$ are defined by

$$
\left.\begin{array}{l}
\mathcal{P}=\left\{[\mu]_{r} U k^{2}-T k^{4}\right\} / \mu(0), \quad q=-\mu_{r} k / \mu(0) \leq 0, \\
r=\left\{-[\mu]_{l} U k^{2}+S k^{4}\right\} / \mu(-L), \quad s=\mu_{l} k / \mu(-L) \geq 0,
\end{array}\right\}
$$

where $[\mu]_{r}=\left(\mu_{r}-\mu(0)\right)$ and $[\mu]_{l}=\left(\mu(-L)-\mu_{l}\right)$. It is worth noting that

$$
\mathcal{P} \geq 0 \quad \text { for } \quad k^{2} \leq k_{1}^{2}=[\mu]_{r} U / T, \quad \text { and } \quad r \leq 0 \text { for } k^{2} \leq k_{2}^{2}=[\mu]_{l} U / S \text {. }
$$

All these equations are in dimensional form. In (2.9), $T$ is the surface tension at the interface $x=0$ and $S$ is the surface tension at the interface $x=-L$. Note that the formulation here allows jumps in viscosities across the interfaces. 
3. Numerical approximation. The problem (2.8) is one-dimensional involving only the independent variable $x$. We use a finite difference discretization of system (2.8) on $(M-1)$ equidistant interior points in the segment $[-L, 0]: x_{M}=-L<x_{M-1}<x_{M-2}<$ $\cdots<x_{1}<x_{0}=0$ and let $d=\left(x_{i}-x_{i+1}\right)$. Then we have the unknowns $f_{0}, f_{1}, f_{2}, \ldots, f_{M}$ and $h_{1}, h_{2}, \ldots ., h_{M-1}$, where $f_{i}=f\left(x_{i}\right)$ and $h_{i}=h\left(x_{i}\right)$.

Using first-order approximation for derivatives at the end points in the boundary conditions $(2.8)_{3}$ and $(2.8)_{4}$, we obtain after some simplifications,

$$
\begin{gathered}
\left(\frac{1}{d \mathcal{P}}-\frac{q}{\mathcal{P}}\right) f_{0}-\frac{1}{d \mathcal{P}} f_{1}=\lambda f_{0}, \\
\frac{1}{d r} f_{M-1}-\left(\frac{1}{d r}+\frac{s}{r}\right) f_{M}=\lambda f_{M} .
\end{gathered}
$$

For interior points we use the central finite difference approximations to the derivatives such as

$$
h_{x}(x)=\frac{h(x+d / 2)-h(x-d / 2)}{d}, \quad h_{x x}(x)=\frac{h(x+d)-2 h(x)+h(x-d)}{d^{2}} .
$$

Using the above finite difference approximations, the discretized form of the system (2.8) becomes

$$
\begin{gathered}
A_{00} f_{0}+A_{01} f_{1}=\lambda f_{0}, \\
A_{i j} f_{j}=-k^{2} U h_{i}, \forall i \in[1, M-1], \forall j \in[0, M], \\
A_{M, M-1} f_{M-1}+A_{M M} f_{M}=\lambda f_{M}, \\
\left\{\eta B_{i m}-\left(\sigma+k^{2} \eta\right) \delta_{i m}\right\} h_{m}=a f_{i}, \forall i, m \in[1, M-1] .
\end{gathered}
$$

This system is overall first-order accurate due to first-order approximations (3.1) and (3.2) at the boundary. This makes the analysis and the results that we obtain below possible. In the above expressions, $A_{i j}$ and $B_{i j}$ are the conventional notation for the entries of the tridiagonal matrices $\mathbf{A} \in \mathbb{R}^{(M+1) \times(M+1)}$ and $\mathbf{B} \in \mathbb{R}^{(M-1) \times(M-1)}$, respectively, which are given below. Above and below, $A_{i, i-1}$ means the entry $A_{i j}$ when $j=i-1$. A similar result holds for the entries of the matrix $\mathbf{B}$ :

$$
\begin{gathered}
A_{00}=\left(\frac{1}{d \mathcal{P}}-\frac{q}{\mathcal{P}}\right), \quad A_{01}=-\frac{1}{d \mathcal{P}}, A_{0 j}=0, \forall j \in[2, M] \\
A_{i, i-1}=-\frac{\mu_{i-1 / 2}}{d^{2}}, A_{i i}=\mu_{i} k^{2}+\frac{\mu_{i-1 / 2}+\mu_{i+1 / 2}}{d^{2}}, A_{i, i+1}=-\frac{\mu_{i+1 / 2}}{d^{2}}, 1 \leq i \leq M-1, \\
A_{M j}=0, \forall j<M-1, A_{M, M-1}=\frac{1}{d r}, A_{M M}=-\left(\frac{1}{d r}+\frac{s}{r}\right) \\
A_{i j}=B_{i, j}=0 \forall j \notin[i-1, i+1], 1 \leq i \leq M-1 \\
B_{i i}=-\frac{2}{d^{2}}, B_{i-1, i}=B_{i, i+1}=\frac{1}{d^{2}}, 1 \leq i \leq M-1
\end{gathered}
$$

We obtain only one relation from (3.4) and (3.6) as follows. From (3.6) we have:

$$
h_{i}=-\frac{a}{\sigma+k^{2} \eta} f_{i}+\frac{\eta}{\sigma+k^{2} \eta} B_{i m} h_{m}, \quad \forall i \in[1, M-1] .
$$


We use the above expression in (3.4) and get

$$
A_{i j} f_{j}=-k^{2} U h_{i}=\left(-k^{2} U\right)\left\{\frac{-a}{\sigma+k^{2} \eta} f_{i}+\frac{\eta}{\sigma+k^{2} \eta} B_{i m} h_{m}\right\}, \quad \forall i \in[1, M-1],
$$

where Einstein summation is implied for the subscripts $m \in[1, M-1]$ and $j \in[0, M]$. We replace again $h_{m}$ by the expression (3.12) obtained from (3.4), in the last term of the right-hand side of the above formula (3.13). The final form of our discretized system (3.3)-(3.6) is given by the following set of equations:

$$
\begin{gathered}
A_{00} f_{0}+A_{01} f_{1}=\lambda f_{0}, \\
A_{i j} f_{j}=\frac{a k^{2} U}{\sigma+k^{2} \eta} f_{i}+\frac{\eta}{\sigma+k^{2} \eta} B_{i m} A_{m j} f_{j}, \\
A_{M, M-1} f_{M-1}+A_{M M} f_{M}=\lambda f_{M},
\end{gathered}
$$

where we recall that $1 \leq i, m \leq(M-1)$ and $0 \leq j \leq M$ for the system (3.15).

4. Approximate estimate of the growth rate. We let $\sigma=\sigma_{R}+i \sigma_{I}$ and $\lambda=$ $\lambda_{R}+i \lambda_{I}, \sigma_{R}, \sigma_{I}, \lambda_{R}, \lambda_{I} \in \mathbf{R}$. Below, we obtain an estimate for the real part $\left(\sigma_{R}\right)$ of the growth rate. We consider (3.15) in the form (Einstein summation in (3.15) is replaced by conventional summation sign)

$$
\left(\sigma+k^{2} \eta\right) \sum_{j=0}^{M} A_{i j} f_{j}=\left(a k^{2} U\right) f_{i}+\eta \sum_{m=1}^{M-1} B_{i m} \sum_{j=0}^{M} A_{m j} f_{j} ; \quad i=1, \ldots, M-1 .
$$

We use the following notation (with the slight abuse of notation because $y$ below has nothing to do with the $y$-coordinate of the setup (see Fig. 1)):

$$
y_{i}=\sum_{j=0}^{j=M} A_{i j} f_{j}, \quad \text { for } i=1, \ldots,(M-1) .
$$

Therefore, the system (4.1) becomes

$$
\left(\sigma+k^{2} \eta\right) y_{i}=a k^{2} U f_{i}+\eta \sum_{m=1}^{M-1} B_{i m} y_{m} .
$$

Let $y^{*}=\max \left\{\left|y_{i}\right|, \quad i=1,2, \ldots,(M-1)\right\}$. The following possibilities exist:

$$
\begin{gathered}
y^{*}=\left|y_{1}\right| \Rightarrow\left|\sigma+k^{2} \eta-\eta B_{11}\right| \leq a k^{2} U \frac{\left|f_{1}\right|}{\left|y_{1}\right|}+\eta\left|B_{12}\right| \\
y^{*}=\left|y_{j}\right|, 1<j<(M-1) \Rightarrow\left|\sigma+k^{2} \eta-\eta B_{j j}\right| \leq a k^{2} U \frac{\left|f_{j}\right|}{\left|y_{j}\right|}+\eta\left(\left|B_{j, j-1}\right|+\left|B_{j, j+1}\right|\right) ; \\
y^{*}=\left|y_{n}\right|, n=(M-1) \Rightarrow\left|\sigma+k^{2} \eta-\eta B_{n n}\right| \leq a k^{2} U \frac{\left|f_{n}\right|}{\left|y_{n}\right|}+\eta\left|B_{n, n-1}\right|
\end{gathered}
$$

We recall that $k, B, \eta$ are real numbers. Then we have the following inequality:

$$
\sigma_{R}+k^{2} \eta-\eta B_{m m} \leq\left|\sigma+k^{2} \eta-\eta B_{m m}\right| .
$$


In the relations (4.4), (4.5), and (4.6) we use the following relations:

$$
\begin{aligned}
B_{11}+\left|B_{12}\right| & =-2 / d^{2}+1 / d^{2} \leq 0, \\
B_{j j}+\left|B_{j, j-1}\right|+\left|B_{j, j+1}\right| & =-2 / d^{2}+1 / d^{2}+1 / d^{2}=0, \quad 1<j<M-1, \\
B_{M-1, M-1}+\left|B_{M-1, M-2}\right| & =-2 / d^{2}+1 / d^{2} \leq 0 .
\end{aligned}
$$

From these inequalities and the relations (4.4)-(4.6), it follows that there exists an integer $p, 1 \leq p \leq(M-1)$, such that $\left|y_{p}\right|=\max _{j}\left\{\left|y_{j}\right|, 1 \leq j \leq M-1\right\}$ (see (4.2)) for which

$$
\sigma_{R}+k^{2} \eta \leq a k^{2} U \frac{\left|f_{p}\right|}{\left|y_{p}\right|} .
$$

In the following, we obtain an estimate of the ratio $\left|f_{p}\right| /\left|y_{p}\right|$. For this, we first need to rewrite the system (3.4) in a suitable form because the matrix with entries $A_{i j}, i=$ $1, \ldots,(M-1) ; j=0,1, \ldots, M$ of the system (3.4) is not a square matrix and hence not invertible. The following subsection discusses how to convert system (3.4) into a wellposed system. In particular, below we convert the nonsquare matrix associated with system (3.4) into a square matrix and then prove that this square matrix is invertible.

4.1. A well-posed system. In order to have only $(M-1)$ unknowns in the new system, we need to first express $f_{0}$ in terms of $f_{1}, f_{2}$ and express $f_{M}$ in terms of $f_{M-1}, f_{M-2}$. Below we use the boundary condition $(2.8)_{5}$ for $h$ and the equation $(2.8)_{1}$ to obtain a new form of the system (3.4) where only the unknowns $f_{1}, f_{2}, \ldots, f_{M-1}$ appear. Then we prove that the matrix of this new system is invertible.

The functions $h$ and $f$ are smooth, and we consider that (2.8) 1 holds also for $x=0$ and $x=x_{M}$. Then we have

$$
\begin{gathered}
-\left.\left(\mu f_{x}\right)_{x}(x)\right|_{x=0}+k^{2} \mu(0) f(0)=0, \\
-\left.\left(\mu f_{x}\right)_{x}(x)\right|_{x=x_{M}}+k^{2} \mu\left(x_{M}\right) f\left(x_{M}\right)=0 .
\end{gathered}
$$

We approximate the derivatives in (4.8) by a finite-difference formula:

$$
\begin{gathered}
\left(\mu f_{x}\right)_{x}(0) \approx\left\{\left(\mu f_{x}\right)\left(x_{0}\right)-\left(\mu f_{x}\right)\left(x_{1}\right)\right\} / d, \\
f_{x}\left(x_{i}\right) \approx\left\{f\left(x_{i}\right)-f\left(x_{i+1}\right)\right\} / d .
\end{gathered}
$$

Then from (4.8) we obtain

$$
f_{0}=-\frac{\mu_{0}+\mu_{1}}{\mu_{0}\left(k^{2} d^{2}-1\right)} f_{1}+\frac{\mu_{1}}{\mu_{0}\left(k^{2} d^{2}-1\right)} f_{2} .
$$

Similar expressions are used to approximate the derivatives in (4.9). Below we use the notation $n=M-1$ for ease of presentation and obtain

$$
f_{n+1}=\frac{\mu_{n}+\mu_{n+1}}{\mu_{n+1}\left(1-d^{2} k^{2}\right)} f_{n}-\frac{\mu_{n}}{\mu_{n+1}\left(1-d^{2} k^{2}\right)} f_{n-1} .
$$

We consider the first equation of the system (3.4) which after using (3.7) becomes

$$
A_{10} f_{0}+A_{11} f_{1}+A_{12} f_{2}=-k^{2} U h_{1} .
$$


Substituting (4.10) for $f_{0}$ and the values of the coefficients $A_{10}, A_{11}$ and $A_{12}$ from (3.8) in the above equation, we obtain after some simplification

$$
\begin{aligned}
& \left\{\frac{-\mu_{1 / 2}}{d^{2}\left(1-k^{2} d^{2}\right)}\left(1+\frac{\mu_{1}}{\mu_{0}}\right)+\frac{\mu_{1 / 2}+\mu_{3 / 2}}{d^{2}}+k^{2} \mu_{1}\right\} f_{1} \\
& +\left\{\frac{\mu_{1 / 2} \mu_{1}}{d^{2} \mu_{0}\left(1-k^{2} d^{2}\right)}-\frac{\mu_{3 / 2}}{d^{2}}\right\} f_{2}=-k^{2} U h_{1} .
\end{aligned}
$$

We consider also the last equation of the system (3.4):

$$
A_{n, n-1} f_{n-1}+A_{n, n} f_{n}+A_{n, n+1} f_{n+1}=-k^{2} U h_{n}, \quad n=M-1 .
$$

We use (4.11) to replace $f_{n+1}$ in the above system in terms of $f_{n}, f_{n-1}$ and obtain

$$
\begin{array}{r}
\left\{\frac{\mu_{n-1 / 2}+\mu_{n+1 / 2}}{d^{2}}+k^{2} \mu_{n}-\frac{\mu_{n+1 / 2}}{d^{2}\left(1-k^{2} d^{2}\right)}\left(1+\frac{\mu_{n}}{\mu_{n+1}}\right)\right\} f_{n} \\
+\left\{-\frac{\mu_{n-1 / 2}}{d^{2}}+\frac{\mu_{n} \mu_{n+1 / 2}}{d^{2} \mu_{n+1}\left(1-k^{2} d^{2}\right)}\right\} f_{n-1}=-k^{2} U h_{n} .
\end{array}
$$

If we let $k d \ll 1$, then

$$
\left(1-k^{2} d^{2}\right) \approx 1
$$

Therefore the denominators in the formula (4.12)-(4.13) are not zero and the expressions (4.12) and (4.13) simplify to

$$
\left\{\frac{-\mu_{1 / 2} \mu_{1}+\mu_{0} \mu_{3 / 2}}{d^{2} \mu_{0}}+k^{2} \mu_{1}\right\} f_{1}+\left\{\frac{\mu_{1 / 2} \mu_{1}-\mu_{0} \mu_{3 / 2}}{d^{2} \mu_{0}}\right\} f_{2}=-k^{2} U h_{1}
$$

and

$$
\left\{\frac{\mu_{n+1 / 2} \mu_{n}-\mu_{n+1} \mu_{n-1 / 2}}{d^{2} \mu_{n+1}}\right\} f_{n-1}+\left\{\frac{\mu_{n+1} \mu_{n-1 / 2}-\mu_{n+1 / 2} \mu_{n}}{d^{2} \mu_{n+1}}+k^{2} \mu_{n}\right\} f_{n}=-k^{2} U h_{n} .
$$

Once the first and the last equations of the system (3.4) are replaced by (4.15) and (4.16), the linear system (3.4) contains only the unknowns $f_{1}, f_{2}, \ldots, f_{M-1}$ and have a square matrix whose entries, now denoted by $A_{i j}^{\prime}, i, j=1,2, \ldots,(M-1)$, are given by

$$
\begin{gathered}
A_{11}^{\prime}=\frac{-\mu_{1 / 2} \mu_{1}+\mu_{0} \mu_{3 / 2}}{d^{2} \mu_{0}}+k^{2} \mu_{1}, A_{12}^{\prime}=\frac{\mu_{1 / 2} \mu_{1}-\mu_{0} \mu_{3 / 2}}{d^{2} \mu_{0}}, A_{1 j}^{\prime}=A_{1 j}, j=3, \ldots, M-1, \\
A_{i j}^{\prime}=A_{i, j}, \quad i=2, \ldots, M-2, \quad j=1, \ldots, M-1, \\
A_{n n}^{\prime}=\frac{\mu_{n+1} \mu_{n-1 / 2}-\mu_{n+1 / 2} \mu_{n}}{d^{2} \mu_{n+1}}+k^{2} \mu_{n}, n=M-1, \\
A_{n, n-1}^{\prime}=\frac{\mu_{n+1 / 2} \mu_{n}-\mu_{n+1} \mu_{n-1 / 2}}{d^{2} \mu_{n+1}}, n=M-1, \\
\left.A_{M-1, j}^{\prime}=A_{M-1, j}, \quad j=1, \ldots, M-3.18 .19\right)
\end{gathered}
$$

Note that the equations corresponding to $i=2, \ldots, M-2$ of the initial system (3.4) remain the same. 
It is easily verified that $\left|A_{i i}^{\prime}\right|>\sum_{j \neq i}\left|A_{i j}^{\prime}\right|$ for each $i \in[2,(M-2)]$. If we have the same relation also for $i=1$ and $i=M-1$, then we can conclude that matrix $\mathbf{A}^{\prime}$ with entries $A_{i j}^{\prime}, i, j=1,2, \ldots, M-1$ is diagonally dominant and hence $A^{\prime}$ is an invertible matrix. Therefore we have to prove that $\left|A_{11}^{\prime}\right|>\left|A_{12}^{\prime}\right|$ and $\left|A_{M-1, M-1}^{\prime}\right|>\left|A_{M-1, M-2}^{\prime}\right|$, where the elements of $A^{\prime}$ are defined in (4.17) and (4.20). That means we need to prove

$$
\left|\frac{-\mu_{1 / 2} \mu_{1}+\mu_{0} \mu_{3 / 2}}{d^{2} \mu_{0}}+k^{2} \mu_{1}\right|>\left|\frac{\mu_{1 / 2} \mu_{1}-\mu_{0} \mu_{3 / 2}}{d^{2} \mu_{0}}\right|
$$

and

$$
\left|\frac{\mu_{n+1} \mu_{n-1 / 2}-\mu_{n+1 / 2} \mu_{n}}{d^{2} \mu_{n+1}}+k^{2} \mu_{n}\right|>\left|\frac{\mu_{n+1 / 2} \mu_{n}-\mu_{n+1} \mu_{n-1 / 2}}{d^{2} \mu_{n+1}}\right| .
$$

Recall that $\mu(x)=a x+b$, which defines $a=\left[\mu_{0}-\mu(-L)\right] / L, b=\mu_{0}$. Hence

$$
\begin{gathered}
\mu_{1 / 2}=b-a d / 2, \quad \mu_{1}=b-a d, \quad \mu_{3 / 2}=b-3 a d / 2, \\
\mu_{n}=a x_{n}+b, \quad \mu_{n+1}=a x_{n+1}+b, \quad \mu_{n-1 / 2}=a x_{n-1 / 2}+b, \quad \mu_{n+1 / 2}=a x_{n+1 / 2}+b .
\end{gathered}
$$

Therefore

$$
\frac{-\mu_{1 / 2} \mu_{1}+\mu_{0} \mu_{3 / 2}}{d^{2} \mu_{0}}=-\frac{a^{2}}{2 \mu_{0}}
$$

and

$$
\frac{\mu_{n+1} \mu_{n-1 / 2}-\mu_{n+1 / 2} \mu_{n}}{d^{2} \mu_{n+1}}=-\frac{a^{2}}{2 \mu_{n+1}} .
$$

We consider in (4.21) and (4.22) a large value of $k$ and a small value of $a$ to have

$$
k^{2} \mu_{1}-\frac{a^{2}}{2 \mu_{0}}>0, k^{2} \mu_{n}-\frac{a^{2}}{2 \mu_{n+1}}>0 .
$$

Consequently, the inequalities (4.21) and (4.22) are equivalent with

$$
k^{2} \mu_{1}>\frac{a^{2}}{\mu_{0}}, k^{2} \mu_{n}>\frac{a^{2}}{\mu_{n+1}} .
$$

The above inequalities hold for large enough $l$ and $k$. Then we obtain $\left|A_{11}^{\prime}\right|>\left|A_{12}^{\prime}\right|$, $\left|A_{M-1, M-1}^{\prime}\right|>\left|A_{M-1, M-2}^{\prime}\right|$ and the matrix $\mathbf{A}^{\prime}$ given by (4.17), (4.18) and (4.20) becomes invertible.

4.2. Diffusion enhanced stability bounds on the growth rate. We will be using the inequality (4.7) to obtain a reasonable bound on the growth rate. For this, we first rewrite $y_{i}$, defined in terms of the entries of the matrix $\mathbf{A}$ in (4.2), in terms of the matrix $\mathbf{A}^{\prime}$, which is

$$
y_{i}=\sum_{m=1}^{m=M-1} A_{i m}^{\prime} f_{m}, \quad f_{i}=\sum_{j=1}^{j=M-1}\left(\mathbf{A}^{\prime-1}\right)_{i j} y_{j}, \quad i=1, \ldots, M-1,
$$

where $\left(\mathbf{A}^{\prime-1}\right)$ is the inverse of matrix $\mathbf{A}^{\prime}$. Using (4.24) in the inequality (4.7), it follows that

$$
\sigma_{R}+k^{2} \eta \leq a k^{2} U \frac{\left|f_{p}\right|}{\left|y_{p}\right|}=a k^{2} U \frac{1}{\left|y_{p}\right|} \sum_{j=1}^{M-1}\left|\left(\mathbf{A}^{\prime-1}\right)_{p j} y_{j}\right| \leq a k^{2} U \sum_{j=1}^{M-1}\left|\left(\mathbf{A}^{\prime-1}\right)_{p j}\right| \frac{\left|y_{j}\right|}{\left|y_{p}\right|} .
$$


We recall (see the line preceding (4.7)) that $\left|y_{p}\right|=\max _{j}\left\{\left|y_{j}\right|, 1 \leq j \leq(M-1)\right\}$. Using this in the above inequality for the estimate of the growth rate, we obtain the following estimate:

$$
\sigma_{R}+k^{2} \eta \leq a k^{2} U \sum_{j=1}^{M-1}\left|\left(\mathbf{A}^{\prime-1}\right)_{p j}\right| .
$$

The matrix $\mathbf{A}^{\prime}$ does not depend on $\eta$. Moreover we have $\mathbf{A}^{\prime-1} \approx \mathrm{O}\left(d^{2}\right)$ and $\mathbf{A}^{\prime-1} \approx$ $\mathrm{O}\left(1 / k^{2}\right)$ because $\mathbf{A}^{\prime} \approx \mathrm{O}\left(1 / d^{2}\right)$ and $\mathbf{A}^{\prime} \approx \mathrm{O}\left(k^{2}\right)$. Then from (4.25) we obtain the following conclusion: the real part of the growth rate is negative with strong diffusion.

In the case of very small wavenumbers $k$, we approximate the system (3.15) after neglecting $k^{2}$ as $k$ is very small. Then we obtain from (4.1),

$$
(\sigma / \eta) \sum_{j=0}^{j=M} A_{i j} f_{j}=\sum_{m=1}^{M-1} B_{i m} \sum_{j=0}^{M} A_{m j} f_{j}, \quad i=1, \ldots, M-1,
$$

and $(\sigma / \eta)$ is an eigenvalue of the matrix $B_{i m}$, with eigenvectors $\sum_{j=0}^{j=M} A_{m j} f_{j}$. In this case, we do not need a new system and the initial matrix with entries $A_{i j}$ does not depend on $k$ and $\eta$. Recall the definition (3.11) of the matrix B. The sum of the elements of each line of $B$ is negative, and hence we obtain $\sigma / \eta \leq 0$. This shows diffusive (diffusion of polymer) slowdown of instabilities.

The matrix $\mathbf{A}^{\prime}$ does not depend on the surface tensions $T$ and $S$ (see relations (4.17), (4.18), (4.20) ). Therefore, the upper bound on $\sigma$ given by (4.25) does not depend on $T$ and $S$. This implies that very large values of $\eta$ can stabilize the flow regardless of the values of the surface tensions. Note that this result appears to be stronger than the one obtained analytically by Daripa and Pasa (see [6]), because the term in the bound there responsible for slowdown due to diffusion depends on surface tensions through the functions $f$ and $h$ (see [6]). However, a careful inspection of the results in [6] shows that the results presented in this paper are consistent with the results of 6 .

5. Comparison. An estimate of the upper bound similar to the one given above by (4.7) can be derived from the results we have obtained in [6] using exact (weak formulation) analysis of the exact system (2.8). To see this, recall the following estimates from [6]:

$$
\sigma_{R} \leq \frac{a F_{1}}{\int_{-L}^{0}|h|^{2} d x}-k^{2} \eta \leq \frac{a\left|F_{1}\right|}{\int_{-L}^{0}|h|^{2} d x}-k^{2} \eta
$$

where $\sigma_{R}=\operatorname{real}(\sigma)$ and $F_{1}$ is the real part of the inner product $\int_{-L}^{0} f(-h)^{*} d x$ (see Daripa and Pasa [6]). Since

$$
\left|F_{1}\right| \leq \sqrt{\left(\int_{-L}^{0}|f|^{2} d x\right)\left(\int_{-L}^{0}|h|^{2} d x\right)},
$$


which follows from the Cauchy-Schwarz inequality, we get

$$
\sigma_{R} \leq a \sqrt{\frac{\int_{-L}^{0}|f|^{2} d x}{\int_{-L}^{0}|h|^{2} d x}}-k^{2} \eta .
$$

We use the rectangle rule to approximate the two integrals appearing in (5.1). We have

$$
\begin{aligned}
& \int_{-L}^{0}|f|^{2} d x \approx\left|f_{1}\right|^{2} d+\left|f_{2}\right|^{2} d+\left|f_{3}\right|^{2} d+\cdots+\left|f_{M-1}\right|^{2} d, \\
& \int_{-L}^{0}|h|^{2} d x \approx\left|h_{1}\right|^{2} d+\left|h_{2}\right|^{2} d+\left|h_{3}\right|^{2} d+\cdots+\left|h_{M-1}\right|^{2} d,
\end{aligned}
$$

where $d$ is the discretization step used in section 3 . Therefore we get

$$
\begin{aligned}
\frac{\int_{-L}^{0}|f|^{2} d x}{\int_{-L}^{0}|h|^{2} d x} & \approx \frac{\left|f_{1}\right|^{2} d+\left|f_{2}\right|^{2} d+\left|f_{3}\right|^{2} d+\cdots+\left|f_{M-1}\right|^{2} d}{\left|h_{1}\right|^{2} d+\left|h_{2}\right|^{2} d+\left|h_{3}\right|^{2} d+\cdots+\left|h_{M-1}\right|^{2} d} \\
& \leq \max _{i}\left\{\frac{\left|f_{i}\right|^{2}}{\left|h_{i}\right|^{2}}\right\}, \quad 1 \leq i \leq M-1 .
\end{aligned}
$$

From the relations (4.2) and (3.4) we have $h_{i}=-y_{i} /\left(k^{2} U\right)$. Therefore, from the above formula, it follows that

$$
\sigma_{R} \leq a k^{2} U \max _{i} \frac{\left|f_{i}\right|}{\left|y_{i}\right|}-k^{2} \eta .
$$

This bound, obtained from numerical approximation of the exact formulae of the upper bound derived in [6], is also implied by (4.7). This is shown below. Since $\left|y_{p}\right|=\max _{j}\left|y_{j}\right|$ in (4.7) (see the line before inequality (4.7)), it follows that

$$
\frac{\left|f_{p}\right|}{\left|y_{p}\right|} \leq \max _{i} \frac{\left|f_{i}\right|}{\left|y_{i}\right|}
$$

and hence inequality (4.7) reduces to (5.2). Thus we see that both procedures give the same formula and, in this sense, the presented numerical method of this paper is also convergent.

We want to emphasize that in Daripa and Pasa 6], using a variational formulation, we obtained an exact formula for the upper bound from which we obtain the above estimate (5.2), which is the same estimate derived in this paper using numerical analysis of the discrete approximation of the underlying scheme. Thus the presented numerical scheme is a reasonable candidate for use in the construction of approximate solutions. However, since this estimate is not exclusively in terms of the data of the problem but also depends on the eigenfunction $f$, this formula (5.2) is not very useful for computing exact numerical values of modal upper bounds a priori. In contrast, in addition to the estimate (5.2) or equivalently (4.7), we have another explicit estimate (4.25) which is in terms of the data of the problem. The unknown ratio $\left|f_{i}\right| /\left|y_{l}\right|$ does not appear any more in the formula (4.25). From this, we also see that a large value of the diffusion coefficient has the potential to completely stabilize the flow, a conclusion also reached in Daripa and Pasa 6 .

Exact computation of the upper bound from (5.2) is not possible in the context of this paper since the estimate (5.2) depends on the eigenfunction $f$, which is not known 
until the differential equations modeling this problem are solved computationally, which falls outside the scope of this work. The present work, as stated early in the abstract, is about the establishment of the stabilizing role of diffusion on hydrodynamic instability using a constructive approach, which has been accomplished in this paper. The bound (5.2) on the growth rate is for individual waves due to dependency of the estimate on the wavenumber $k$. We can compute values of the modal upper bound for individual waves from (5.2) (which is implied by (4.7)) provided we assume, for the purpose of exposition of the effect of diffusion on stability, the value of $\min _{i}\left|f_{i}\right| /\left|y_{i}\right|$, where the minimum is taken over all $i$. We plot the modal upper bound against the wavenumber $k$ taking $\min _{i}\left|f_{i}\right| /\left|y_{i}\right|=10 /(a U)$ and $\eta=4$ (recall that $a$ is the slope of the basic profile of the viscosity and $\eta$ is the viscosity coefficient). There is no specific reason behind these numbers. Any other choice will do as well to reveal the qualitative nature of the plots in Fig. 2. Figure 2 shows plots of the upper bound for individual waves against the wavenumber for three cases: (i) the solid curve is for the case with no diffusion; (ii) the dash-and-dotted curve is for the case with diffusion for modest values of the diffusion coefficient $\eta$ (specifically when $a U \frac{\left|f_{i}\right|}{\left|y_{l}\right|}>\eta$ ); (iii) the dashed curve is also for the case with diffusion but for very large values of the diffusion coefficient (specifically when $\left.a U \frac{\left|f_{i}\right|}{\left|y_{l}\right|}<\eta\right)$. These figures show qualitatively the nature of the stabilizing effect of diffusion and in the extreme situation (the dashed curve), the flow can be completely stabilized. In reality, qualitatively in some finite neighborhood of the point $(0,0)$, Fig. 2 should compare well with similar plots obtained from an exact computation of the differential equations.

6. Conclusion. The constructive approach of this paper (see section 4) and the weak formulation approach of Daripa and Pasa (2007) provide similar estimates (4.7) and (5.2), respectively, for the real part of the growth rate. Moreover, the formula (4.25) in this paper and Theorem 1 in Daripa and Pasa (2007) clearly show that species diffusion suppresses instability. For appropriate values of the parameters of our model, a large enough diffusion coefficient can reverse the instability process. As a function of the wavenumbers, the growth rate is bounded by a parabola with a maximum point (with the leading term $-\eta$ ) given by the estimate (4.25). Since the proposed numerical scheme in this paper provides a bound comparable to the exact one, the proposed scheme should be useful to numerically construct approximate solutions and thereby explore the effect of diffusion.

The technique used in this paper is of general interest and very constructive, which leads to implementation of the algorithm. Furthermore, the technique presented here should be applicable in principle to multi-layer Hele-Shaw flows (more than three-layer) and other multi-layer flows involving species diffusion. Such problems are usually governed by a system of advection-diffusion equations, similar to the system of equations (1)-(3). Since such problems are abundant in all areas of classical physics and biomedical sciences, to name just a few, the technique used here should be applicable to many applied problems involving species diffusion. 


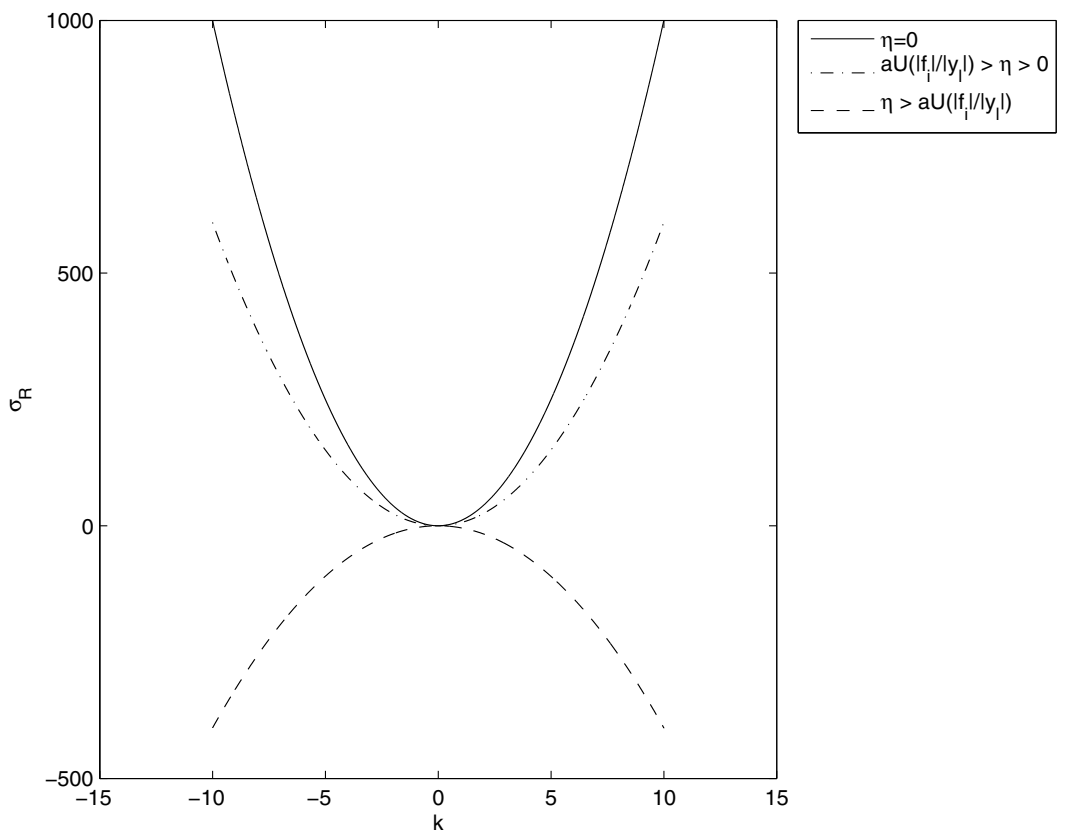

FIG. 2. Stabilizing effect of diffusion: plots of modal upper bound on the growth rate versus wavenumber with and without diffusion. The solid curve is for the case with no diffusion and the other curves for cases with diffusion.

In conclusion, we should mention that diffusive slowdown has been proved in this paper and in our earlier paper [6] considering only normal modes. Without diffusion, the problem is selfadjoint, but with diffusion the problem is not selfadjoint which raises the possibility that in addition to modal behavior, there is the possibility of transient growth due to nonmodal behavior. This issue has not been explored here. Such possiblities of transient growth due to nonmodal behavior in shear flows can be found in the book of Henningson and Schmid 22.

\section{REFERENCES}

[1] R. F. Almgren, Crystalline Saffman-Taylor fingers, SIAM J. Appl. Math. 55, 1511-1535 (1995) MR 1358787 (96g:80009)

[2] R. L. Chouke, P. van Meurs, and C. Van der Poel, The stability of a slow, immiscible, viscous liquid-liquid displacement in a permeable media, Petrol. Trans. AIME. 216, 188-194 (1959)

[3] P. Daripa and G. Pasa, New bounds for stabilizing Hele-Shaw flows, Appl. Math. Lett. 18(11), 1293-1303 (2005) MR2170886 (2006d:76037)

[4] P. Daripa and G. Pasa, On the Growth Rate for Three-Layer Hele-Shaw Flows: Variable and Constant Viscosity Cases, Int. J. Engrg. Sci. 43(11-12), 877-884 (2005) MR2163169(2006c:76040)

[5] P. Daripa and G. Pasa, A simple derivation of an upper bound in the presence of viscosity gradient in three-layer Hele-Shaw flows, J. Stat. Mech. 11 pages, P01014. (2006) doi:10.1088/1742$5468 / 2006 / 01 / \mathrm{P} 01014$

[6] P. Daripa and G. Pasa, Stabilizing Effect of Diffusion in Enhanced Oil Recovery and Three-Layer Hele-Shaw Flows with Viscosity Gradient, Transp. Porous Media 70(1), 11-23 (2007) MR2336218 (2008h:76045) 
[7] J. Escher and G. Simonett, On Hele-Shaw models with surface tension, Math. Res. Lett. 3, 467-474 (1996) MR1406012 (97i:35145)

[8] F. J. Hickernell and Y. C. Yortsos, Linear stability of miscible displacement processes in porous media in the absence of dispersion, Stud. Appl. Math. 74, 93-115 (1986) MR836292 (87e:76073)

[9] G. M. Homsy, Viscous fingering in porous media, Ann. Rev. Fluid Mech. 19, 271-311 (1987)

[10] S. D. Howison, Complex variable methods in Hele-Shaw moving boundary problems, Eur. J. Appl. Math. 3, 209-224 (1992) MR1182213 (94f:76025)

[11] S. B. Gorell and G. M. Homsy, A theory of the optimal policy of oil recovery by the secondary displacement process, SIAM J. Appl. Math. 43, 79-98 (1983) MR687791 (84b:76073)

[12] L. P. Kadanoff, Exact Solutions for the Saffman-Taylor Problem with Surface Tension, Phys. Rev. Letts. 65, 2986-2988 (1990)

[13] D. A. Kessler, J. Koplik, and H. Levine, Pattern selection in fingered growth phenomena, Adv. in Phys. 37, 255-329 (1998)

[14] W. Littman, Polymer Flooding: Developments in Petroleum Science. 24 Elsevier, Amsterdam (1988)

[15] D. Loggia, N. Rakotomalala, D. Salin, and Y. C. Yortsos, The effect of mobility gradients on viscous instabilities in miscible flows in porous media, Phys. Fluids. 11(3), 740-742 (1999)

[16] J. W. McLean and P. G. Saffman, The effect of surface tension on the shape of fingers in a HeleShaw cell, J. Fluid Mech. 102, 445-469 (1981)

[17] R. B. Needham and P. H. Doe, Polymer flooding review, J. Pet. Technol. 12, 1503-1507 (1987)

[18] Q. Nie and F. R. Tian, Singularities in Hele-Shaw flows, SIAM J. Appl. Math. 58, 34-54 (1998) MR 1610037 (2000c:76023)

[19] P. G. Saffman, Exact solutions for the growth of fingers from a flat interface between two fluids in a porous medium or Hele-Shaw cell, Quart. J. Mech. Appl. Math. 12, 146-155 (1959) MR0104422 $(21: 3177)$

[20] P. G. Saffman, Viscous Fingering in Hele-Shaw Cells, J. Fluid Mech. 173, 73-94 (1986) MR877015 $(87 \mathrm{~m}: 76062)$

[21] P. G. Saffman and G. I. Taylor, The penetration of a fluid in a porous medium or Hele-Shaw cell containing a more viscous fluid, Proc. Roy. Soc. A., 245, 312-329 (1958) MR0097227 (20:3697)

[22] P. J. Schmid and D. S. Henningson, Stability and Transition in Shear Flows, Springer-Verlag, New York (2001) MR1801992 (2001j:76039)

[23] D. Shah and R. Schecter, Improved Oil Recovery by Surfactants and Polymer Flooding, Academic Press, New York, 1977

[24] M. Shariati and Y. C. Yortsos, Stability of miscible displacements across stratified porous media, Phys. Fluids 13(8) 2245-2257 (2001)

[25] K. S. Sorbie, Polymer-Improved Oil Recovery. CRC Press, Boca Raton, Florida, 1991

[26] S. Tanveer, Surprises in viscous fingering, J. Fluid Mech. 409, 273-308 (2000) MR1756392 (2002a:76059)

[27] F. R. Tian, A Cauchy integral approach to Hele-Shaw problems with a free boundary: the case of zero surface tension, Arch. Ration. Mech. Anal. 135, 175-196 (1996) MR.1418464 (97j:35167)

[28] A. C. Uzoigwe, F. C. Scanlon, and R. L. Jewett, Improvement in polymer flooding: The programmed slug and the polymer-conserving agent, J. Petrol. Tech. 26, 33-41 (1974)

[29] G. L. Vasconceles and L. P. Kadanoff, Stationary solutions for the Saffman-Taylor problem with surface tension Phys. Rev. A. 44, 6490-6495 (1991)

[30] Y. C. Yortsos and M. Zeybek, Dispersion driven instability in miscible displacement in porousmedia, Phys. Fluids 31, 3511-3518 (1988) 